\title{
Plädoyer für Gründung der Bundespflegekammer
}

\section{Hier steht eine Anzeige.}

— Dr. Markus Mai, Präsident der rheinlandpfälzischen Landespflegekammer, hat sich anlässlich des Deutschen Pflegetags 2017 vehement für die Errichtung einer Bundespflegekammer ausgesprochen. Gemeinsam mit anderen Akteuren der Pflege, wie Andreas Westerfellhaus, Präsident des Deutschen Pflegerats, fordert Mai eine institutionalisierte Interessenvertretung für die Pflegenden auch auf Bundesebene.

„Neben der notwendigen klaren Positionierung in den einzelnen Bundesländern müssen auch zwingend der Bundesgesetzgeber und die Bundesregierung von uns Pflegenden direkt über unsere Forderungen informiert werden. Gerade auf Bundesebene werden viele Weichen für die Pflege, aber auch für das gesamte Gesundheitssystem, gestellt", betonte Mai.

Die Bundespflegekammer werde als Spitzenorganisation der pflegerischen Selbstverwaltung auf Bundesebene das berufliche Zusammengehörigkeitsgefühl der professionell Pflegenden stärken, fachliche und berufspolitische Aktivitäten länderü- bergreifend strategisch ausrichten und die pflegerische Versorgung der Gesellschaft auf nationaler Ebene gestalten. „Wir müssen auch auf Bundesebene dafür sorgen, dass eine sachgerechte und professionelle Pflege der Bevölkerung sichergestellt ist", so Mai.

Für den Gründungsprozess der Bundespflegekammer (in Gründung) soll nach Ansicht der Vertreterversammlung der Kammer in Rheinland-Pfalz eine „Gründungskonferenz zur Errichtung der Bundespflegekammer" mit mindestens den Vertretern der bereits vollständig etablierten Kammern (d.h. in Bundesländern, in denen es bereits Gesetze zur Errichtung von Landespflegekammern gibt) gebildet werden.

Die Bundespflegekammer soll im Laufe der nächsten Legislaturperiode des Bundestages (2017-2021) abschließend gegründet werden und ihre Funktion aufnehmen. Dies wäre ein entscheidendes Signal an die Bundespolitik, heißt es.

www.bundespflegekammer.de

\section{ZQP \\ Neue Datenbank zur Pflegeforschung}

— Das Zentrum für Qualität in der Pflege (ZQP) hat eine neue, frei zugängliche und deutschlandweit einmalige Online-Datenbank mit aktuell über 900 Forschungsprojekten veröffentlicht. Diese ermöglicht eine gezielte Recherche wissenschaftlicher Studien der letzten zwölf Jahre. Damit bietet sie sowohl für die Pflegeforschung als auch die Pflegepraxis ein Angebot, das inhaltlich beispielsweise von Wohn- und Versorgungsformen über pflegerische Interventionen und Konzepte, Beratung bis hin zur pflegerischen Bildung, Gesundheitsförderung, Prävention, Rehabilitation und E-Health reicht. Die Datenbank hilft, Schwerpunkte in Deutschland zu erkennen, Forschungslücken und Förderer zu identifizieren oder einfach aktuelle Projekte aufzufinden. Sie bietet vor allem auch Instituten und Forschungsgruppen die Chance, eigenen Arbeiten noch mehr Sichtbarkeit zu verschaffen. Gelistet sind laufende sowie bereits abgeschlossene Projekte, die in einem fast zweijährigen Verfahren durch das ZQP systematisch online recherchiert sowie qualitätsgesichert aufbereitet wurden. An die Erstrecherche der Daten hat sich ein fortlaufender Prüfzyklus angeschlossen. In diesem wird regelmäßig nach Aktivitäten von Einrichtungen mit pflegewissenschaftlicher Ausrichtung sowie von wissenschaftlichen Institutionen gesucht, die sich mit themenrelevanten Forschungsprojekten etabliert haben. Ergänzend erfolgt eine kontinuierliche Recherche in Fachzeitschriften und Literaturdatenbanken.

www.zqp.de/forschungsdatenbank 